\title{
Corrigendum
}

\section{Corrigendum to "Identification of Tomato Disease Types and Detection of Infected Areas Based on Deep Convolutional Neural Networks and Object Detection Techniques"}

\author{
Qimei Wang, ${ }^{1}$ Feng Qi ${ }^{1},{ }^{1}$ Minghe Sun $\mathbb{D},{ }^{2}$ Jianhua Qu ${ }^{1},{ }^{1}$ and Jie Xue ${ }^{1}$ \\ ${ }^{1}$ Business School, Shandong Normal University, Jinan, Shandong, China \\ ${ }^{2}$ University of Texas at San Antonio, San Antonio, TX, USA \\ Correspondence should be addressed to Feng Qi; cliff@sdnu.edu.cn
}

Received 1 July 2020; Accepted 1 July 2020; Published 9 February 2021

Copyright (C) 2021 Qimei Wang et al. This is an open access article distributed under the Creative Commons Attribution License, which permits unrestricted use, distribution, and reproduction in any medium, provided the original work is properly cited.

In the article titled "Identification of Tomato Disease Types and Detection of Infected Areas Based on Deep Convolutional Neural Networks and Object Detection Techniques" [1], there was an error in the corresponding author affiliation, where the author Feng Qi's affiliation "University of Texas at San Antonio, San Antonio, Texas, USA" should be corrected to "Business School, Shandong Normal University, Jinan, Shandong, China."

The corrected corresponding author's affiliation is shown in the author information above.

\section{References}

[1] Q. Wang, F. Qi, M. Sun, J. Qu, and J. Xue, "Identification of Tomato Disease Types and Detection of Infected Areas Based on Deep Convolutional Neural Networks and Object Detection Techniques," Computational Intelligence and Neuroscience, vol. 2019, Article ID 9142753, 15 pages, 2019. 